passed from the globe to the outside of the enclosing hemispheres, their interior being perfectly devoid thereof. This distribution of electricity to the outside of conducting bodies was further illustrated by placing within each other three metallic goblets or cells, each a size smaller than the other, and insulated from each other by a cake of shellac. On the application of the electric fluid to the inside of the innermost cell, it was found, as indicated by the electrometer, to pass instantaneously to the outer surface of the largest cell. The same result attended a somewhat similar experiment made with a piece of wire gauze, made into the shape of a vessel, like the wire gauze portion of the Davy lamp. The electric fluid in this instance also passed at once to the outside of the vessel. We may mention that Dr. Faraday stated in the theatre, that this last-named experiment he had noticed previously, but he had little doubt of the result.

Induction takes place through the air and through all other bodies, conductors as well as insulators. In metals, which have the power of conduction, care must be that they are not touched, so that their conducting power come not into play. Sulphur possesses very great power of induction.

Then comes the question, as to the state of our globe as regards electricity. Electrical phenomena have ever been looked upon as caused by the vicissitudes of the weather, the changes of temperature, \&c.; but Dr. Faraday regards as the most important phenomenon of electricity, the growth of plants, by which they discharge the electric fluid from the atmosphere, and consequently act as lightning conductors. It is a far more important phenomenon than thunder-storms. Peltier advanced a startling theory with respect to the electrical condition of the globe. He thought the globe was in a highly negative state, and the surrounding atmosphere the reverse-that is, highly positive; in other words, he believed all matter to be negatively electrified, all space positively so. Without canvassing this theory, Dr. Faraday is inclined to believe that the sun, planets, \&c., are in a state of electricity opposite to that of the earth, and are sustained thus by the power of induction. That the inhabitants of the earth cannot tell whether it is or is not in a highly electrified condition by walking on it, was shown by connecting a large metallic plate, with the electrical machine, and placing a white mouse on it. Although the plate was high electrified, as was demonstrated by repeated discharges, it had not any effect on the little animal, even when the discharge took place very near it.

With respect to thunder-storms:-The clear high air, which can be electrified, is a non-conductor; but if a cloud forms it conducts the electric fluid, as does moist air, from particle to particle, to the outer surface of the cloud in a general mass. A cloud consists of thousands of little particles or vesicles, all of them conductors of electricity. Dr. Faraday remarked that he could not clearly understand how a highly electrified cloud coheres, it being the first principle of electricity to repel particles similarly electrified. The particles of the cloud are most likely held together by the induction of the earth. Every particle which is electrified acts towards the external surface, even those which arein the interior of the cloud. In this way may be explained the intensity of a flash of lightning.

\section{ROYAL MEDICAL AND CHIRURGICAL} SOCIETY.

Tuesday, January 10, 1843.

Dr. Williams, President.

Case of Obscure Disease in the Chest. By C. J. Graham Tice, M.D., Assistant Surgeon 8th Reg. of Foot. [Communicated by Sir J. M'Grigor, Bart.]

The patient in this case was the quarter-master of the regiment, a man of plethoric and nervous temperament, aged forty-eight. On the 7 th of September, 1842 , he complained of pain in the left side, slightly affecting the respiration; pressure over the liver caused uneasiness; he had a disagreeable taste in the mouth like fried onions. He was treated with calomel and purgatives, and lost blood from the arm, but with only temporary relief. The dyspnœa increased in severity, and he had much cough, and the only easy position in which he could lie was leaning forward holding both knees. Percussion elicited no morbid sound, and auscultation proved that the lungs on both sides, as well as the heart, were healthy. Active treatment, consisting of venesection, calomel, antimony, \&c., was pursued without relief. On the 8th of October he was salivated. Up to this time the cough had been dry, now there was abundant expectoration of glairy viscid mucus. Notwithstanding the severity of the symptoms, the pulse was little affected. At this time Dr. William Stokes saw the patient, and, after careful examination, pronounced the lungs and heart to be healthy. He considered the disease depending on nervous and spasmodic irritation of the respiratory organs, and advised expectorants and sedatives. Soon after this the breathing became stridulous and the cough laryngeal; there was a distressing sense of choking, and the same fœtor of the breath. No relief followed the treatment adopted-viz., leeches and extract of belladonna, externally; and calomel, tartar emetic, and sedatives, internally. The stridulous breathing was now replaced by mucous râle and a total absence of vesicular breathing on the right side, and nearly so on the left. He died suddenly shortly after this, six weeks from the commencement of his illness.

Examination of the Body Thirty Hours after Death. Both lungs were healthy, save being a little gorged with blood; on separating the right lung the knife entered a cavity the size of a pullet's egg, from which a most offensive odor was emitted. It was formed by an abscess in a mass of enlarged bronchial glands at the bifurcation of the trachea, and opening into the right bronchus. A loose calcareous mass was partially engaged in the lower part of the bronchus, almost wholly obstructing its canal. The mucous membrane of both bronchi, above the ulceration, was marked by a deep blush. A small ulcer was found towards the inner edge of the descending cornu of the thyroid cartilage on the right side. The remains of the suppurative glandular mass lay in the bifurcation of the trachea, pressing on the glandular surface of the œsophagus. The author remarks that the appearances on dissection satisfactorily explain the phenomena observed during life. The fœtid taste in the mouth arose from the abscess opening into the bronchial tubes. The sense of suffocation, no doubt, was caused by the pressure of the large tumor on the trachea; and 
the sudden extinction of life may be accounted for by the calcarcous mass falling into the right bronchus, added to the partial obliteration of the left.

On the Decrement of Weight in Phthisis. By RoBert Williaxs, M.D., Physician to St. Thomas's Hospital.

The author states, that some years ago he began a series of experiments on possible remedies in phthisis, satisfied that general treatment was of little avail, and that the cure of the disease must be sought for in a specific remedy. This series embraced preparations of platina, palladium, ormium, iridium, tilanium, chromium, and cerium. He subsequently tried every seed that Messrs. Charlwood, of Covent-garden, could furnish, and he had previously tried every wood, every bark, and every gum he could obtain. Nothing ap. peared beneficially to influence the disease; the result was, as usual, uniformly fatal, but the termination was not accelerated as to time, or aggravated in the preceding phenomena. The pathological appearances, also, in the cases examined after death, were the same as when the ordinary form of treatment was adopted; not the slightest attempt at reparation was seen in any part of the lungs. No injury, however, was done, except in two cases treated with white hellebore, in both of which death was so remarkably hastened that it seemed as if that substance, or probably the veratrine it is said to contain, acted as a poison in phthisis. In making these experiments the author determined on weighing the patients, considering that an increase or decrease of weight would afford a better criterion of amendment, or otherwise, than the fallacious hopes with which nature cheers the individual in this desolating disease.

The numbers weighed were few, perhaps eight, unexpected difficulties having occurred and the experiments being prematurely terminated in some cases by the patient leaving the hospital. One general law, however, was observed in all-viz., that the loss of weight was not continued, but intermittent; or, the patient being weighed weekly, and, as nearly as possible, under the same circumstances, showed an alternate increment and decrement generally of one or more pounds on each alternate week. The decrement, however, usually exceeded the increment, and consequently every few days an increasing balance was left against the patient. Several cases were related illustrative of the law, which the author states does not appear to have been mentioned by any writer; these, however, our limits will not allow us to give. The following are the concluding remarks of the author:"The large number of substances I have tried as possible remedies for the cure of this fatal disorder has as. sured me that there is no class of substances which a prudent physician, beginning with small doses, and gradually increasing them, may not safely make use of in his attempts to cure this or any other equally intractable disorder; and as experiment is the only means by which medicinc can be advanced, I strongly recommend the adoption of this practice, at least in public hospitals, as an imperative duty. The only inference which I am enabled to deduce from the singular law of the alternate increment and decrement of weight in phthisis, is that it may afford an explanation of the buoyant fcelings of the patient, who must necessarily feel his symptoms ameliorated, and his health improved every few days. It is evidently the measure of the last flickerings of the vital principle, but how the lamp of life is fed is, perhaps, bcyond our power of explanation."

\section{SHEFFIELD MEDICAL SOCIETY.}

Jan. 13, 1813.

The President in the Chair.

Dr. Favell exhibited the following morbid specimens :-

I. A Portion of Lung thickly studded with Tubercles, in a state of softening, and a small Cavity at the Apex.-The patient had presented the usual general and physical signs of phthisis, but had died very suddenly. There was nothing in the chest or abdomen to account for the suddenness of his death. No cerebral symptoms were present, and the brain was not examined. The upper lobe of each lung presented a sinilar morbid appearance, except that the only cavity was at the apex of the left.

II. Lamelliform Ossification of the Pleura.-The patient was a young man who died of phthisis. On examining the chest there was manifest depression in the subclavicular portion on each side. It was motionless during the act of respiration, and yielded an extremely dull sound on percussion. The adhesion between the pleuræ on the right side was remarkably firm, but on the left it was found almost impossible to effect a separation. When the left lung was removed, the pleura covering it was observed to be in three different morbid states-part of it was considerably thickened, but not otherwise altered in character, part was cartilaginous, and a large mass covering the upper lobe was converted into perfect bone.

III. An Enlarged Heart, on the surface of which were several irregular patches of partially organised lymph.-The patient had not suffered from any affection of the pericardium, whilst he had been under Dr. Favell's care, but the Doctor exhibited the specimen as one which showed the pre-existence of partial pericarditis-a form of disease which all pathologists do not admit.

IV. Nutmeg Liver-Hypertrophy of the White Substance.-The patient was a young man, aged twenty-six, and had been employed as a butcher's laborer. He had led an irregular life, and been much addicted to drinking. The tissue of the liver was harder than natural, and there was slight effusion into the abdominal cavity. Tubercles existed in the lungs.

V. A Portion of Lung containing a Scirrhous Tubercle about twice the size of a common hazel nut, taken from the body of a female who died of cancer uteri.-During the latter period of her life she suffered considerably from pulmonary irritation.

VI. A Scirrhous Uterus with its Appendages.-The uterus was of about the normal size, but its texture extremely hard. The os uteri was entirely destroyed. The ovaries were greatly enlarged and indurated ; the right contained a quantity of semi-gelatinous fluid. Neither the bladder nor rectum were involved in the disease, but a large scirrhous tubercle was formed upon the meso-rectum. The lining membrane of 